\title{
Cosmic matter in the laboratory - the Compressed Baryonic Mat- ter experiment at FAIR
}

\author{
Peter Senger ${ }^{1, a}$ \\ ${ }^{1}$ GSI Helmholtzzentrum für Schwerionenforschung GmbH, Planckstr. 1, 64291 Darmstadt, Germany
}

\begin{abstract}
The Compressed Baryonic Matter (CBM) experiment will be one of the major scientific pillars of the future Facility for Antiproton and Ion Research (FAIR) in Darmstadt. The goal of the CBM research program is to explore the QCD phase diagram in the region of high baryon densities using high-energy nucleus-nucleus collisions. This includes the study of the equation-of-state of nuclear matter at neutron star core densities, and the search for the deconfinement and chiral phase transitions. The CBM detector is designed to measure rare diagnostic probes such as hadrons including multi-strange (anti-) hyperons, lepton pairs, and charmed particles with unprecedented precision and statistics. Most of these particles will be studied for the first time in the FAIR energy range. In order to achieve the required precision, the measurements will be performed at very high reaction rates of 1 to $10 \mathrm{MHz}$. This requires very fast and radiation-hard detectors, a novel data read-out and analysis concept based on free streaming front-end electronics, and a high-performance computing cluster for online event selection. The physics program and the status of the proposed CBM experiment will be discussed.
\end{abstract}

\section{Introduction}

The experimental and theoretical investigation of the properties of elementary matter under extreme conditions is a major topic of international fundamental research. Experiments with high-energy heavy-ion collisions are performed worldwide at the large accelerator facilities, in order to create and to study extremely hot and dense matter in the laboratory. In heavy-ion collisions at very high beam energies, as provided by the Relativistic Heavy Ion Collider at BNL in USA or by the Large Hadron Collider at CERN in Switzerland, elementary matter at extremely high temperatures is created, more than hundred-thousand times hotter than the core of our sun. Under such conditions, a plasma is created which consists of quarks and gluons. This plasma is a mixture of elementary matter and antimatter, similar to the primordial soup in early universe about a microsecond after the big bang. In heavy-ion collisions lower bombarding energies, as available at the SPS at CERN and at the future Facility for Antiproton and Ion Research (FAIR) at Darmstadt, nuclear matter is strongly compressed. The matter density in the collision zone of two heavy nuclei exceeds more than five times the density of an atomic nucleus. In nature, such densities are expected to exist in the core of a neutron star.

The common goal of these experiments is to explore the various forms and phases of stronglyinteracting matter. Similar to water, which exhibits different states separated by phase transitions,

a e-mail: p.senger@gsi.de 
it is expected that the degrees of freedom of nuclear matter change with temperature and density, thereby creating novel forms of elementary matter. The fundamental theory of strong interaction, Quantum Chromo Dynamics (QCD), predicts a smooth crossover transition from hadronic matter to the quark-gluon plasma at a pseudo-critical temperature of about $160 \mathrm{MeV}$ for vanishing netbaryon density [1-3]. However, QCD still fails to make predictions about the properties of matter at high net-baryon densities. Our conception of compressed nuclear matter is based on effectivemodel calculations which predict structures in the QCD phase diagram at high net-baryon densities, like a critical endpoint followed by a first-order phase transition, or new forms of exotic matter [4-6]. Figure 1 illustrates the conjectured phases of nuclear matter together with the locations of cosmic matter in a diagram of temperature versus net-baryon density.

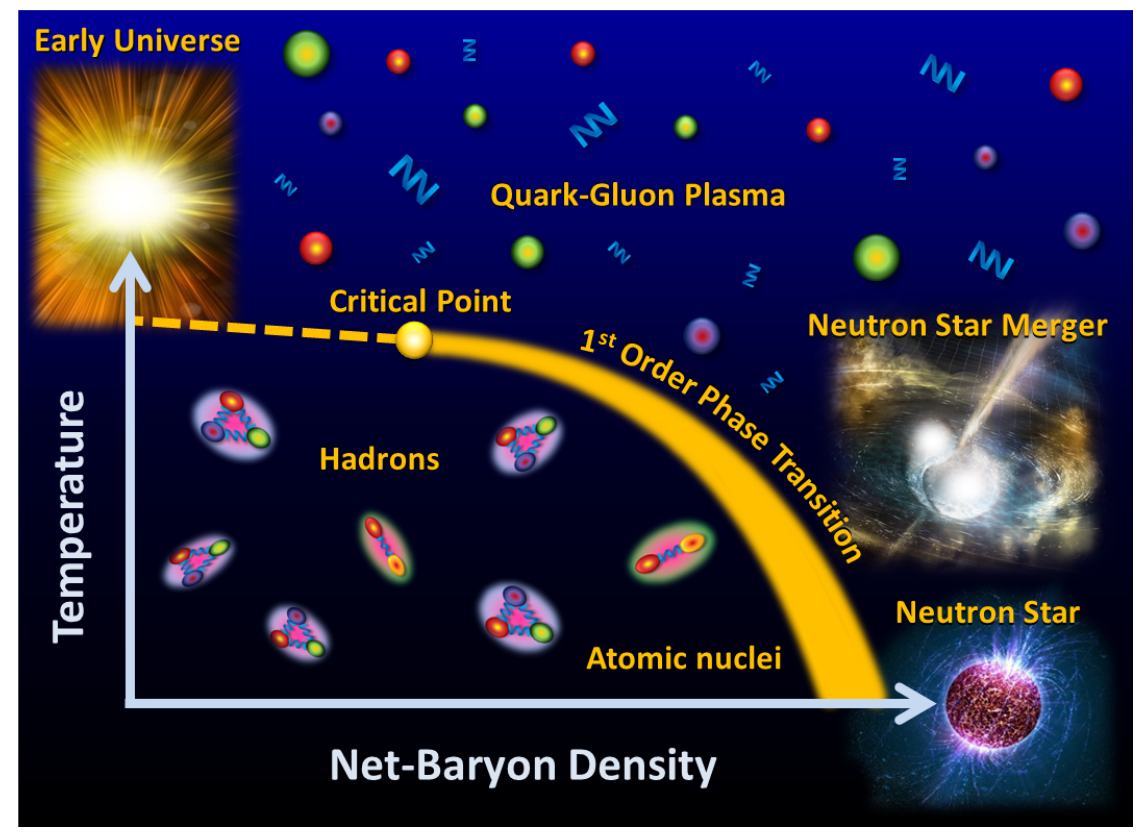

Figure 1. Sketch of the phase diagram of strongly interacting matter with conjectured structures and locations of cosmic matter. In the early universe, the temperature was very high, but the net-baryon density was zero. In contrast, the net-baryon density in neutron star cores is very high, about 5 times the density of atomic nuclei, but the temperature is very low. The matter in neutron star mergers is also highly compressed but much hotter than in neutron stars, similar to the matter created in a heavy-ion collision at FAIR energies.

Experimentally, only little is known about the QCD phase diagram. The comparison of the measured yields of particles and antiparticles to the results of thermal model calculations provides a so called freeze-out temperature as function of baryon chemical potential [7,8]. This temperature is measured at a late stage of the collision, when the produced particles cease to interact, and the density has dropped well below saturation density. However, for LHC and top RHIC energies, the freeze-out temperature coincides with the pseudo-critical temperature predicted by QCD, indicating that freezeout and hadronization might happen simultaneously. The mission of heavy-ion experiments at lower beam energies includes the search for the landmarks in the QCD phase diagram, such as the critical point and a first order phase transition. Another fundamental ingredient for our understanding of nu- 
clear matter is its equation-of-state, which describes the matter properties as function of pressure and density, and, determines the evolution of supernova explosions, the size and radius of neutron stars, and the dynamics of neutron star mergers.

Moreover, heavy-ion collision experiments offer the unique opportunity to experimentally address an-other fundamental question: why is the mass of protons and neutrons about 50 times larger than the sum of the masses of their building blocks, the up and down quarks? The mass of the nucleons, and, hence, the mass of the visible universe, is not explained by the Higgs mechanism. According to our present knowledge, the kinetic energy of quarks and gluons essentially contributes to the nucleon mass. If nucleons dissolve in hot and /or dense matter, they should not only lose their identity, but also their mass. The discovery of such a Chiral phase transition would be a breakthrough in our understanding of the origin of the mass of the visible universe.

\section{The CBM Physics Program at SIS100}

FAIR will provide heavy-ion beam energies from $2 \mathrm{~A}$ - $11 \mathrm{~A} \mathrm{GeV}$ for $\mathrm{Au}$ or $\mathrm{U}$ nuclei, up to $14 \mathrm{~A} \mathrm{GeV}$ for $\mathrm{N}=\mathrm{Z}$ nuclei, and up to $29 \mathrm{GeV}$ for protons with the SIS100 synchrotron. Already in central $\mathrm{Au}+\mathrm{Au}$ collisions at $5 \mathrm{~A} \mathrm{GeV}$ the nuclear fireball will be compressed - according to transport model and hydro calculations [9] - to more than 6 times saturation density $\rho_{0}$, and at $10 \mathrm{~A} \mathrm{GeV}$ even a density above 8 $\rho_{0}$ is reached as illustrated in figure 2. At such densities, the nucleons will start to melt and to dissolve into their constituents. The calculations predict that the dense fireball spends a relatively long time within the phase coexistence region or even beyond.
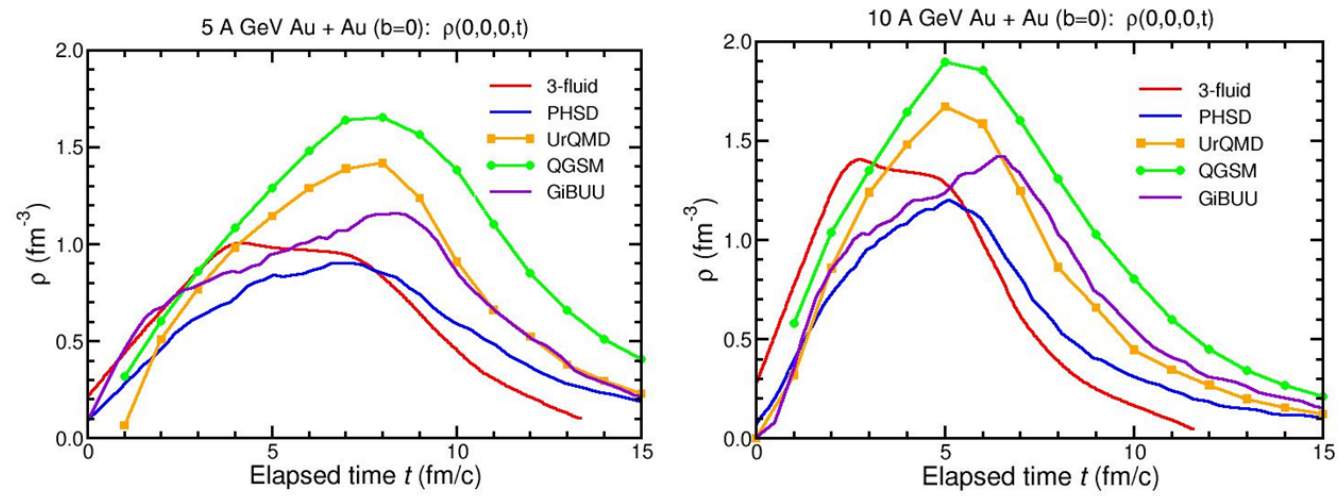

Figure 2. Evolution of the central net baryon density $\rho(t)$ as function of elapsed time as calculated by different transport models and by a 3-fluid hydrodynamics code for central $\mathrm{Au}+\mathrm{Au}$ collision at $5 \mathrm{~A} \mathrm{GeV}$ (left panel) and at $10 \mathrm{~A} \mathrm{GeV}$ (right panel)[9].

Further indication, that a phase transition might occur at densities reached at SIS100 beam energies, comes from a non-local 3-flavor Nambu Jona-Lasinio model calculation of a neutron star, which predicts the development of a mixed phase of hadrons and quarks above densities of about $5 \rho_{0}$, and the transition to pure quark matter above $8 \rho_{0}$ [10]. The results of this calculation, which is able to reproduce a 2 solar mass neutron star, is depicted in figure 3 . 


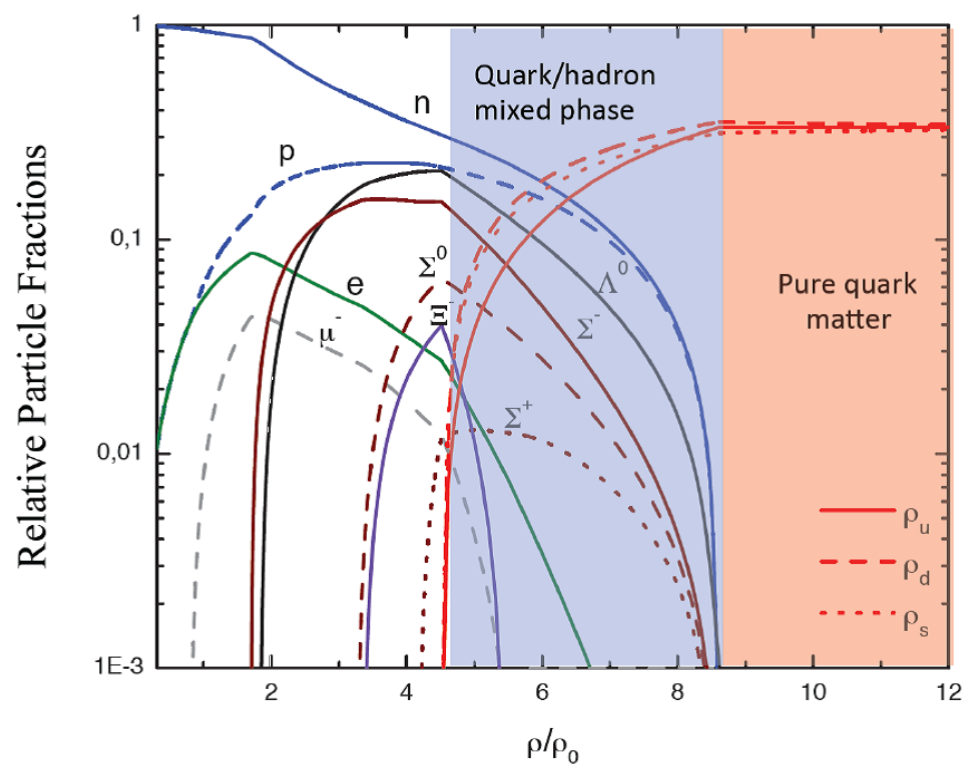

Figure 3. Particle population in a neutron star calculated with a Nambu Jona-Lasinio (n3NJL) model with repulsive vector interactions. The model is able to describe neutron stars with 2 solar masses and radii between 12 and $13 \mathrm{~km}[10]$.

In conclusion, the beam energies available at SIS100 appear to be especially well suited for generating signals of the phase transition, and, therefore, offer the opportunity to address fundamental scientific questions as mentioned above. The focus of the CBM experiment at FAIR is to study messengers from the dense fireball such as multiple strange hyperons, lepton pairs, and hadrons containing charm quarks in order to find answers to the questions raised above. A survey of the theoretical concepts and the experimental programs devoted to the exploration of the QCD phase diagram with focus on high baryon densities is given in the CBM Physics Book [11]. The CBM research program at SIS100 includes the physics cases and observables as discussed in the following.

\subsection{The equation of state of nuclear matter at high baryon densities}

According to transport models, multi-strange (anti-)hyperons are produced in sequential collisions involving kaons and Lambda hyperons, and, therefore, are sensitive to the density in the fireball. This sensitivity is expected to increase towards lower beam energies close to or even below the production threshold. The excitation functions of multi-strange hyperons $\left(\Xi^{-}(d s s)\right.$ and $\left.\Omega^{-}(s s s)\right)$ and anti-hyperons $\left(\Xi^{+}(\bar{d} \bar{s} \bar{s})\right.$ and $\left.\Omega^{+}(\bar{s} \bar{s} \bar{s})\right)$ in A+A collisions with different A values at SIS100 beam energies are very promising observables which will shed light on the matter equation of state at neutron star core densities. Another promising observable is the collective flow of identified particles which is driven by the pressure gradient inside the fireball, and hence, is expected to be sensitive to the compressibility of nuclear matter. Up to now, only the proton flow excitation function has been measured at the AGS [12], and was used to extract the compressibility of nuclear matter [13]. 


\subsection{Phase Transitions, phase coexistence, critical point}

The experimental observation, that in ultra-relativistic heavy-ion collisions multi-strange hyperons including $\Omega^{-}$and $\Omega^{+}$are in chemical equilibrium like all the other produced particles, was taken as strong indication that the system had undergone a transition from a partonic phase to the hadronic final state, with the equilibration being driven by multi-body collisions in the high particle density regime near the phase boundary [14]. Agreement of the hyperon yield with thermal model calculations was found also at $40 \mathrm{~A} \mathrm{GeV}$ in $\mathrm{Pb}+\mathrm{Pb}$ collisions at the SPS [15]. In $\mathrm{Ar}+\mathrm{KCl}$ collisions at an energy of $1.76 \mathrm{~A} \mathrm{GeV}$, however, the measured yield of $\Xi^{-}$hyperons exceeds the thermal model prediction by about a factor of 20, indicating that $\Xi^{-}$hyperons are far off chemical equilibrium [16]. High precision measurements of excitation functions of multi-strange hyperons in A+A collision at SIS100 energies will allow to study the degree of equilibration of the fireball, and, hence, open the possibility to find a signal for the onset of deconfinement in QCD matter at high net-baryon densities.

The slope of the dilepton invariant mass spectrum between 1 and about $2.5 \mathrm{GeV} / c^{2}$ reflects the average temperature of the fireball [17]. The precise measurement of the spectral slope as a function of beam energy opens the unique possibility to measure the caloric curve, which would be the first direct experimental signature for phase coexistence in high-density nuclear matter. This measurement would also provide indications for the onset of deconfinement and the location of the critical endpoint. Another direct experimental proof for a first order phase transition would be the discovery of phase coexistence by observing an enhanced production of composite particles or multi-particle correlations caused by the spinodal amplification of density fluctuations [18].

In classical binary liquids, density fluctuations grow when approaching the critical point, leading to critical opalescence. A similar phenomenon is predicted in heavy-ion collisions, namely event-byevent fluctuations of conserved quantities such as baryon number, strangeness and electrical charge, which can be related to thermodynamical susceptibilities. Lattice QCD calculations suggest, that higher moments of these distributions are sensitive to vicinity of the critical point. Recent measurements by the STAR collaboration provide evidence for an increase of higher-order fluctuations towards lower beam energies [19]. Corresponding data in the FAIR energy range are required in order to search for a peak of these fluctuations, as expected for a critical behavior.

\subsection{In-medium modifications of hadrons, onset of chiral symmetry restoration}

Lepton pairs will be measured over a wide range of invariant masses, starting from low-mass vector mesons including their Dalitz decays up to charmonium. The precise measurement of lepton pairs at low invariant masses will allow to analyze modifications of vector meson properties in dense baryonic matter. This observable is expected to be sensitive to chiral symmetry restoration [20]. The thermal radiation at intermediate invariant dilepton masses includes a broadened in-medium $\rho$ meson, radiation from the QGP, and dileptons from multi-pion annihilation. The latter contribution reflects $\rho-a_{1}$ chiral mixing, and, therefore, provides a direct link to chiral symmetry restoration.

\subsection{Charm}

With CBM at SIS100, charm production will be studied for the first time at beam energies close to production threshold. At these energies, the formation time of charmonium is small compared to the lifetime of the reaction system. CBM is thus uniquely suited to study the interactions between fully formed $J / \psi$ and the dense medium with appropriate counting statistics and systematics. Systematic measurements of charmonium in $\mathrm{p}+\mathrm{A}$ collisions with varying target mass number $\mathrm{A}$ at proton energies up to $29 \mathrm{GeV}$ will shed light on the charmonium interaction with cold nuclear matter, and constitute 
an important baseline for measurements in heavy-ion collisions. Moreover, the simultaneous measurement of open charm will give access to the basically unknown charm production cross section at or near the kinematic threshold. According to a recent UrQMD calculation the subthreshold charm production in central $\mathrm{Au}+\mathrm{Au}$ collision increased dramatically when considering secondary processes like $N^{*} \rightarrow \Lambda_{c}+D$ and $N^{*} \rightarrow N+J / \psi[21]$.

\subsection{Hypernuclei and strange objects}

Thermal models predict a maximum in the production probability of light hypernuclei in heavy-ion collisions at SIS100 beam energies [22,23]. The CBM experiment is well suited to measure hydrogen and helium hypernuclei, including light double-lambda hypernuclei. The discovery of (double-) lambda hypernuclei and the determination of their lifetimes will provide valuable information on the hyperon-nucleon and hyperon-hyperon interactions, which are essential ingredients for the understanding of the nuclear matter equation-of-state at high densities, and, hence, of the structure of neuron stars [24].

\section{Experimental challenge}

Most of the diagnostic probes discussed above are rarely produced at beam energies where the highest baryon densities will be created. Therefore, the key feature of existing and future experiments is rate capability in order to measure these observables with unprecedented precision. The experimental challenge is to combine a large-acceptance and fast detector setup with a high-speed data read-out system and a high luminosity beam. Several experiments concentrate on the exploration of the QCD phase diagram at in the region of high baryon densities. Explorative heavy-ion experiments at AGS in Brookhaven [12] and CERN-SPS [25] mainly measured abundantly produced hadrons. The NA61/SHINE experiment at CERN-SPS continues to scan the QCD phase diagram light and medium size beams [26]. The STAR collaboration at RHIC plans for a second beam energy scan to improve the statistical significance of the data taken in the first series of measurements [27]. In order to go down in collision energy and up in baryon-chemical potential, the STAR detector can be operated in a fixed target mode. The HADES collaboration investigates the production of dileptons and (strange) hadrons in collisions of beams of pions, protons, and nuclei with nuclear targets at GSI SIS18 [28]. At the Joint Institute for Nuclear Research (JINR) in Dubna, the collider facility NICA together with a multi-purpose detector is planned at JINR [29]. As an intermediate step, a fixed target experiment called "Baryonic Matter at Nuclotron" (BM@N) is being installed at the Nuclotron to study heavy-ion collisions at gold-beam energies up to about $4.5 \mathrm{~A} \mathrm{GeV}$. The luminosity limitations of these existing and future facilities constrain the research programs to the investigation of bulk observables, and prevent high precision measurements of rare diagnostic probes. In contrast, the Compressed Baryonic Matter (CBM) experiment at the Facility for Antiproton and Ion Research (FAIR) is designed to run at extremely high interaction rates (up to $10 \mathrm{MHz}$ ). This feature is the key requirement for the measurement of multi-differential observables including rare probes with unprecedented precision. The rate capabilities of existing and planned heavy-ion experiments are plotted in fig. 4 as function of beam energy. The comparison clearly demonstrates the uniqueness of the CBM setup in terms of rate capability. 


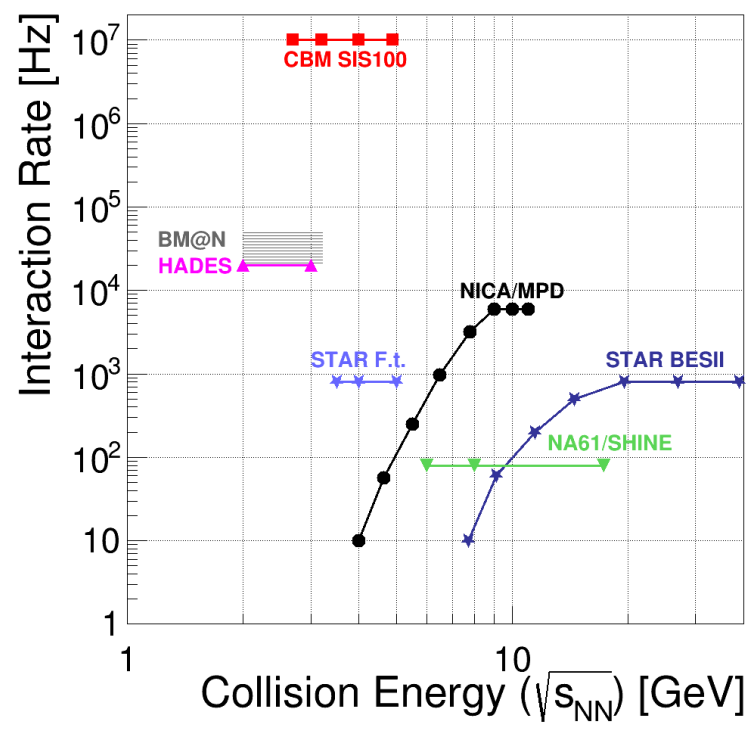

Figure 4. Interaction rates achieved by existing and planned heavy-ion experiments as function of beam energy (taken from [30]).

\section{The Compressed Baryonic Matter (CBM) Experiment}

The research program on dense QCD matter at FAIR will be performed by the experiments CBM and HADES which are shown in figure 5. The two setups will be operated alternatively. The HADES detector, with its large polar angle acceptance ranging from 18 to 85 degrees, is well suited for reference measurements with proton beams and heavy-ion collision systems with moderate particle multiplicities, such as $\mathrm{Ni}+\mathrm{Ni}$ or $\mathrm{Ag}+\mathrm{Ag}$ at low SIS100 energies. With the HADES detector, electron-positron pairs and hadrons including multi-strange hyperons can be reconstructed.

The CBM setup is a fixed target experiment covering polar emission angles between 2.5 and 25 degrees. The detector system has been designed as a multipurpose device which will be capable to measure hadrons, electrons and muons in elementary nucleon and heavy-ion collisions over the full FAIR beam energy range [11]. For particle identification several detectors are needed. The masses of hadrons which traverse the full setup (protons, pions, kaons) are determined by the measurement of their momentum and velocity. The particle momentum is derived from the curved trajectories in a magnetic field. The particle trajectories are measured by the Silicon Tracking System (STS) based on double-sided silicon micro-strip sensors arranged in eight stations inside a dipole magnet. In order to reduce multiple scattering, the sensors are read out via low-mass micro cables by free-streaming ASICs which are mounted on frontend boards outside the active detector area. The superconducting dipole magnet has a gap between the pole shoes of $1.44 \mathrm{~m}$ height and a field integral of $1 \mathrm{Tm}$. The particle time-of-flight is measured by a wall of Multi-Gap Resistive Plate Chambers (MRPC) with low resistivity glass electrodes providing a time resolution of $50 \mathrm{ps}$ at rates up to $25 \mathrm{kHz} / \mathrm{cm}^{2}$. The MRPC wall has an active area of $100 \mathrm{~m}^{2}$ and is located about $7 \mathrm{~m}$ downstream the target. Both centrality and reaction plane angle of the collision will be measured with the Project Spectator Detector, which is a segmented hadronic calorimeter located about 10 me downstream the target. 
Short-lived hadrons (e.g. the hyperons $\Lambda, \Xi, \Omega$ ) and hypernuclei decaying inside the magnetic field are identified by their invariant mass which is reconstructed via the measured trajectories and the time-of-flight of their decay products. In this case, the decay vertex has to be determined accurately by the STS. Even hyperons with one neutral daughter (e.g. $\Sigma^{+} \rightarrow \mathrm{p}+\pi^{0}$ ) can be identified via the missing mass method. In order to identify open charm hadrons $\left(\Lambda_{c}\right.$, D mesons) with decay lengths between $c \tau=60 \mu \mathrm{m}$ and $312 \mu \mathrm{m}$, the Micro-Vertex-Detector (MVD) is required, which consists of four layers of silicon monolithic active pixel sensors, located between $5 \mathrm{~cm}$ and $20 \mathrm{~cm}$ downstream the target in the vacuum of the beam pipe.

The identification of lepton pairs requires additional detector systems. Electrons and positrons up to momenta of $6 \mathrm{GeV} / \mathrm{c}$ can be separated from pions and heavier hadrons by a Ring Imaging Cherenkov $(\mathrm{RICH})$ detector. The RICH consists of a large volume $\mathrm{CO}_{2}$ radiator, where the high energetic electrons travel faster than light and produce Cherenkov photons, which are measured by a UV photon detector based on multi-anode photomultipliers. Additional pion suppression is achieved in particular for electrons with momenta above $2 \mathrm{GeV} / \mathrm{c}$ - by a four layer Transition Radiation Detector which can be operated at particle densities up to $100 \mathrm{kHz} / \mathrm{cm}^{2}$. Alternative to electrons, also muons will measured by a Muon Chamber (MuCh) system which consists of Gas Electron Multiplier (GEM) detector triplets sandwiched between hadron absorber plates made of graphite and iron. For muon measurements, the RICH detector will be replaced by the MuCh, and the TRD serves as tracking detector after the last hadron absorber.

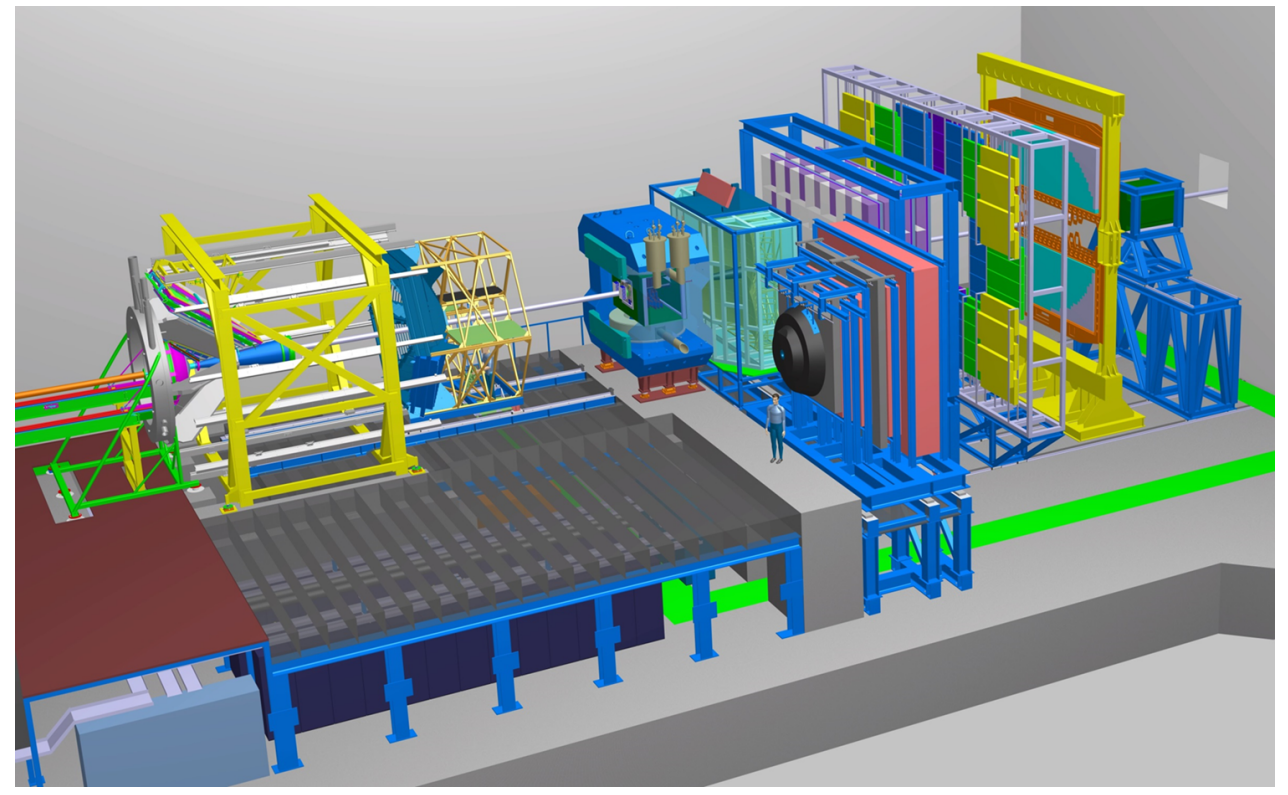

Figure 5. The HADES detector (left) and the CBM experimental setup (right) with Ring Imaging Cherenkov detector in measuring position, and the muon detection system in parking position.

In order to perform high-precision multi-differential measurements of rare probes the experiment should run at event rates of up to $10 \mathrm{MHz}$ for several months per year. To filter out weakly decaying particles like multi-strange hyperons, hypernuclei or D mesons, no simple trigger signal can be generated. Instead, the full events have to be reconstructed, and the decay topology has to be identified 
online by fast algorithms running on a high-performance computing farm hosted by the GSI GreenIT cube. To utilize the maximum rates, the data acquisition is based on self-triggered front-end electronics, which provides a time stamp for each signal. The online track reconstruction algorithms combine detector hits to tracks based on the space and time information of the hits ("4-D" reconstruction). Figure 6 depicts the tracks of charged particles produced in a simulated central collision of two gold nuclei at a beam energy of $10 \mathrm{~A} \mathrm{GeV}$ as reconstructed by the MVD and the STS.

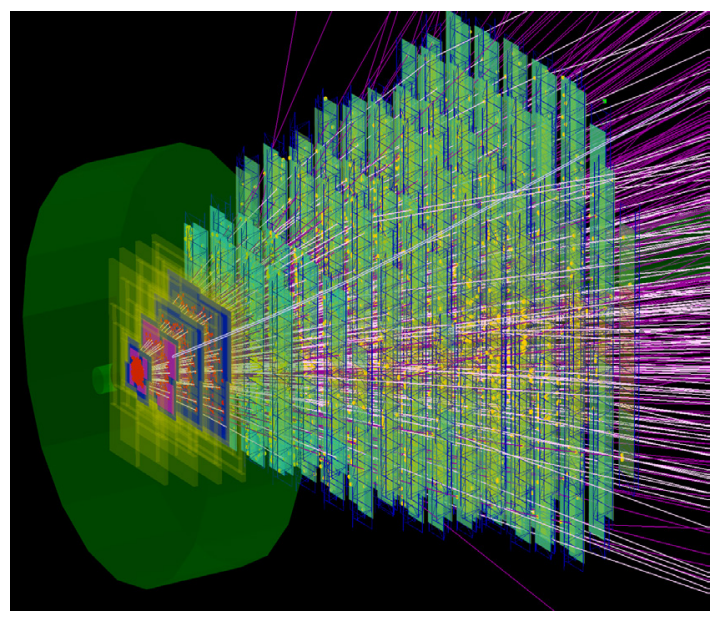

Figure 6. Simulation of a central collision of two gold nuclei at a beam energy of $10 \mathrm{~A} \mathrm{GeV}$. The trajectories of about 700 charged particles including the decay products of short-lived particles will be measured with the CBM silicon tracking detectors MVD and STS.

The development of the experimental components is well in progress. The Technical Design Reports on the Superconducting Dipole Magnet, on the Silicon Tracking System, on the Ring Imaging Cherenkov Detectors, on the Projectile Spectator Detector, on the Time-of-Flight detector, and on the Muon Chamber system have been approved by FAIR. The TDRs on Data Acquisition and First Level Event Selection, on the Micro-Vertex-Detector, and on the Transition Radiation Detector will be submitted in 2017.

Until the start of FAIR, the nuclear matter research program at GSI will be pursued with the HADES experiment at SIS18. At the SIS100 accelerator, the HADES detector can be used to perform di-electron and hadron reference measurements in collision systems with moderate particle multiplicities, such as proton-proton, proton-nucleus and nucleus-nucleus collisions with light nuclei.

\section{Summary}

The Compressed Baryonic Matter (CBM) experiment will be one of the major scientific pillars of the future Facility for Antiproton and Ion Research (FAIR) in Darmstadt. The goal of the CBM research program is to explore the QCD phase diagram in the region of high baryon densities using high-energy nucleus-nucleus collisions. This includes the study of the equation-of-state of nuclear matter at neutron star core densities, and the search for the deconfinement and chiral phase transitions. The CBM detector is designed to measure rare diagnostic probes such as multi-strange hyperons, charmed particles and vector mesons decaying into lepton pairs with unprecedented precision and 
statistics. Most of these particles will be studied for the first time in the FAIR energy range. In order to achieve the required precision, the measurements will be performed at reaction rates between 100 $\mathrm{kHz}$ and $10 \mathrm{MHz}$. This requires very fast and radiation hard detectors, and a novel data read-out and analysis concept based on free streaming front-end electronics and a high-performance computing cluster for online event selection. The use of the most modern detector and computer technology is the prerequisite for a substantial discovery potential of heavy-ion collision experiments at FAIR energies.

\section{Acknowledgments}

The design and development of the CBM experiment is performed by the CBM Collaboration which actually consists of more than 470 persons from 56 institutions and 11 countries. The CBM project is supported by the German Ministry of Education and Research, the Helmholtz Association, and national funds of the CBM member institutions.

\section{References}

\section{References}

[1] S. Borsanyi et al., JHEP 1009 (2010) 073

[2] A. Basavov et al.: Phys. Rev. D85 (2012) 054503

[3] Y. Aoki et al., Nature 443 (2006) 675

[4] J. Luecker et al., arXiv:1308.4509v1 [hep-ph]

[5] L. McLerran and R. D. Pisarski, Nucl. Phys. A796, 83-100 (2007),

[6] K. Fukushima and T. Hatsuda: Rept. Prog. Phys. 74 (2011) 014001

[7] F. Becattini et al., Phys. Rev. Lett. 111 (2013) 082302

[8] A. Andronic et al., arXiv:1611.01347 [nucl-th]

[9] I.C. Arsene et al., Phys. Rev. C75 (2007) 24902

[10] M. Orsaria et al., Phys. Rev. C89 (2014)015806

[11] The CBM Physics Book, B. Friman et al.(eds): Series: Lecture Notes in Physics, Vol. 814 (2011)

[12] C. Pinkenburg et al., Phys. Rev. Lett. 83 (1999) 1295

[13] P. Danielewicz et al., Science 298 (2002) 1592

[14] P. Braun-Munzinger, J. Stachel and C. Wetterich, Phys.Lett. B 596 (2004) 61

[15] A. Andronic, P. Braun-Munzinger and J. Stachel, Acta Phys. Polon. B 40 (2009) 1005

[16] G. Agakishiev et al. (HADES collaboration),arXiv:1512.07070v1

[17] R. Rapp and H. van Hees, Phys. Lett. B 753 (2016) 586

[18] J. Steinheimer and J. Randrup, Phys. Rev. Lett. 109 (2012) 212301

[19] L. Adamczyk et al., Phys. Rev. Lett. 113 (2014) 092301

[20] P. Hohler and R. Rapp, Phys. Lett. B731 (2014) 103-109

[21] J. Steinheimer, A. Botvina, M. Bleicher, arXiv:1605.03439v1

[22] A. Andronic et al., Phys. Lett. B697 (2011) 203

[23] H. Stöcker et al., Nucl. Phys. A 827 (2009) 624c

[24] A. S. Botvina et al., Phys. Lett. B 742 (2014) 7

[25] C. Alt et al., Phys. Rev. C77 (2008)024903

[26] A. Laszlo, Pos(CPOD07)054 (2007)

[27] https://drupal.star.bnl.gov/STAR/starnotes/public/sn0598 
[28] G. Agakishiev et al., (HADES Collaboration), Eur. Phys. J. 41 (2009) 243

[29] D. Blaschke et al. (eds), Eur. Phys. J. A, 528 (2016) 267

[30] T. Ablyazimov et al., [CBM Collaboration], Eur. Phys. J. A 53 (2017) 60 